\title{
Optical observations of GRB afterglows: GRB 970508 and GRB 980326 revisited $^{\star, \star \star}$
}

\author{
A.J. Castro-Tirado ${ }^{1,2}$ and J. Gorosabel ${ }^{1}$ \\ 1 Laboratorio de Astrofísica Espacial y Física Fundamental (LAEFF-INTA), P.O. Box 50727, E-28080 Madrid, Spain \\ ${ }^{2}$ Instituto de Astrofísica de Andalucía (IAA-CSIC), P.O. Box 03004, E-18080 Granada, Spain
}

Received January 21; accepted March 17, 1999

\begin{abstract}
Since January 1997, we have monitored 15 GRB fields, detecting 6 optical/IR afterglows. We have revisited GRB 970508 and GRB 980326. For GRB 970508, we derive a power-law decay exponent $\alpha=-1.19$ ( $R$-band). The luminosity of the host galaxy $L$ relative to the characteristic luminosity $L^{\star}$ is in the range $0.06-0.15$, i.e. a dwarf galaxy. For GRB 980326, we derive a power-law decay exponent $\alpha=-1.7$, taking into account the new upper limit for the host as $R \geq 27.3$ provided by Bloom \& Kulkarni (1998). This implies one of the fastest GRB optical decays ever measured. The fact that only about $50 \%$ of optical transients have been found within the $\gamma / \mathrm{X}$-ray error boxes, suggest that either considerable intrinsic absorption is present or that some optical transients display a very fast decline. We also propose that the "secondary maximum" detected on 17 Apr. 1998 could be explained in the context of the "SN-like" light curves 2-3 weeks after the GRB, as recently suggested by Woosley (1999).
\end{abstract}

Key words: methods: observational — gamma-rays: bursts

\section{Introduction}

Since 1997, we have been monitoring the error boxes of gamma-ray bursts (hereafter GRBs) detected by the BSAX or RXTE satellites. We have monitored 15 GRB fields on the aggregate, detecting 6 optical/IR afterglows. See Castro-Tirado (1999) and Gorosabel (1999) for a review of results. Here we focus on the GRB 970508 and GRB 980326 afterglows.

\section{Observations: Some selected results}

\subsection{GRB 970508 and its host galaxy}

The optical counterpart was discovered by Bond (1997). After an initial phase of constant brightness lasting one

Send offprint requests to: J. Gorosabel (jgu@laeff.esa.es)

* Based on observations collected at the German-Spanish Astronomical Centre, Calar Alto, operated by the Max-PlanckInstitut für Astronomie, Heidelberg, jointly with the Spanish National Commission for Astronomy.

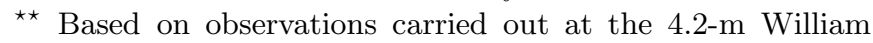
Herschel Telescope operated by the Royal Greenwich Observatory in the Spanish Observatorio del Roque de los Muchachos of the Instituto de Astrofísica de Canarias. day (as inferred from the light curves shown in Fig. 1), a strong flare took place. The peak brightness of the optical light occurred two days after the GRB, and a maximum was reached in the $R$ and $U$ bands at the same time. The increase in brightness was similar to the one inferred for GRB 970228 (Guarnieri et al. 1997).

New $U$ and $R$-band images taken on 11 May showed that the source was declining in brightness (Castro-Tirado et al. 1997) and by ten months later, it almost reached the quiescent level, revealing a 25th mag galaxy in the $R$-band (Bloom et al. 1998a; Castro-Tirado et al. 1998a). The decay of the optical flux could be modelled in terms of a power law decay, with $F_{R} \propto t^{-1.19 \pm 0.01}\left(\chi^{2} /\right.$ dof $\left.=2.56\right)$, assuming a host galaxy with $R=25.72 \pm 0.20$ according to Bloom et al. (1998). On the basis of an extrapolation of the $V$ - and $B$-band measurements into the $U$-band, assuming a given spectral shape (Bloom et al. 1998b; Zharikov et al. 1998), we obtain $U=26.56 \pm 0.25$ for the host. If the $U$-band flux also followed a power-law decay, then $F_{U} \propto t^{-1.69 \pm 0.34}\left(\chi^{2} /\right.$ dof $\left.=2.37\right)$, implying a faster decay than that observed at longer wavelength. This difference in decay slope between $F_{R}$ and $F_{U}$ is marginal given the large error on the $U$-band decay slope.

With respect to the host galaxy, and depending on the value of the $K$-correction and spectral index $\beta\left(F_{\nu} \propto \nu^{-\beta}\right)$ considered, we obtain $M_{B}=-17.53 \pm 0.5(\beta=-3)$ or $M_{B}=-16.6 \pm 0.6(\beta=-1.56)$. The first value is consistent with that reported by Zharikov et al. (1998). In both cases the object is well below the knee of the galaxy luminosity function, $M_{B}^{\star} \sim-20.6$ (Schechter 1976) and the luminosity of the host galaxy $L$ relative to the characteristic luminosity $L^{\star}$ is 0.06 or 0.15 depending of the value of $M_{B}$ considered. The host galaxy corresponds to any of the following categories: a starburst, a red dwarf starburst, an irregular dwarf, a HII galaxy or a blue compact dwarf galaxy. All these types of dwarf galaxies show evidence of starburst or post starburst activity, and are now thought to harbour the majority of star formation at $z \sim 1$

\subsection{GRB 980326}

Optical observations were performed with the $2.2-\mathrm{m}$ CAHA telescope (equipped with CAFOS). See Fig. 2. 


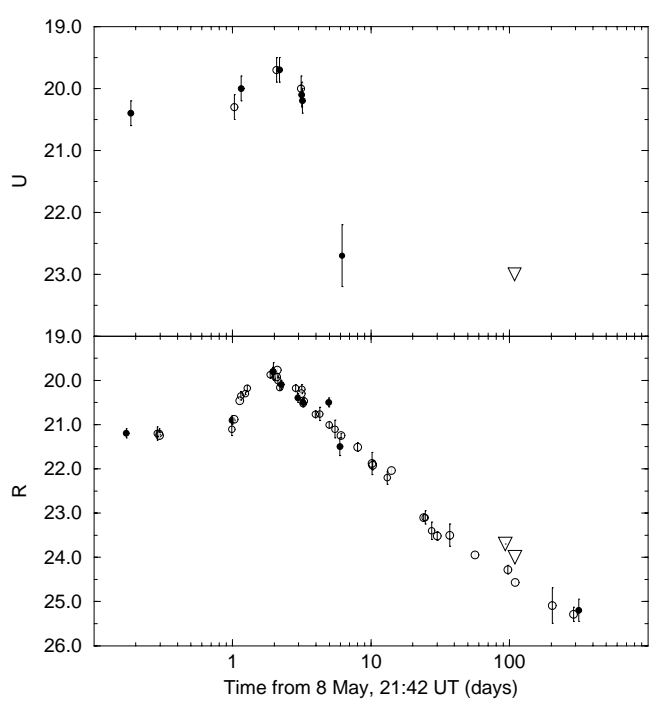

Fig. 1. The $U$ and $R$-band light-curves of the optical transient related to GRB 970508. Filled circles are data based on our observations obtained at the $4.2-\mathrm{m}$ WHT, $2.56-\mathrm{m}$ NOT and 2.2-m CAHA telescopes on 9-15 May, from Castro-Tirado et al. (1998b). Empty circles are data taken from Pedersen et al. (1998); Galama et al. (1998); Bloom et al. (1998a); Zharikov et al. (1998) and diverse IAU circulars. Upside down triangles are upper limits

The resulting 2700-s co-added image revealed an object (2.6 $\sigma$ confidence level), consistent with the position of the optical counterpart discovered by Groot et al. (1998). We derive $R=22.8 \pm 0.7$, in agreement with the measurements obtained by other authors.

If we introduce a host galaxy with $R_{\mathrm{c}}=25.5$ as initially proposed by Djorgovski et al. (1998), then we obtain $F_{R} \propto t^{-2.12 \pm 0.11}$ with $\chi^{2} /$ dof $=1.32$ (point-dashed line in Fig. 2). The value of $\alpha$ (the power-law exponent) is consistent with the one calculated by Groot et al. (1998) as $\alpha=-2.10 \pm 0.13$.

However, the recent upper limit of $R \geq 27.3$ given for the GRB 980326 host galaxy (Bloom \& Kulkarni 1998) implies $F_{R} \propto t^{-1.70 \pm 0.13}$ with $\chi^{2} /$ dof $=3.45$ (long dashed line), instead of $F_{R} \propto t^{-2.12 \pm 0.11}$ when considering a $R_{\mathrm{c}}=25.5$ host. In that case, the light curve can then be interpreted as a power-law decay plus an optical outburst occurring approximately 20 days post-burst, when $R_{\mathrm{c}}=25.5$ was measured for the optical transient at this date. This "secondary maximum" could be explained in the context of the "SN-like" light curves 2-3 weeks after the GRB, as recently suggested by Woosley (1999).

The value derived for the power-law decay exponent $(\alpha=-1.70 \pm 0.13)$ implies one of the fastest optical decay ever detected for a GRB. The existence of similar slopes in the GRB afterglow light curves could explain -at least in some cases- the non-detection of optical counterparts for many events, like GRB 970111, GRB 970616 , GRB 970815 and GRB 970828.

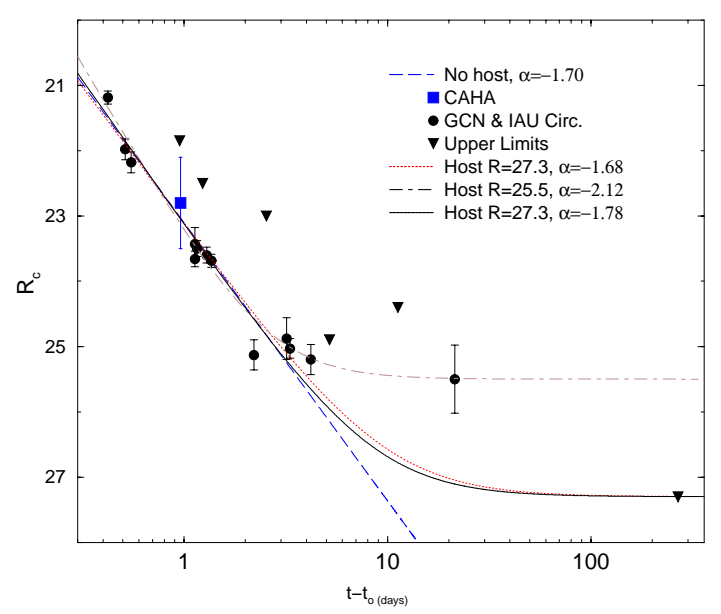

Fig. 2. The $R_{\mathrm{c}}$ band light curve for the GRB 980326 optical counterpart. The circles and triangles represent the measurements and the upper limits taken from the literature (GCN notices, IAU circulars) and from Groot et al. (1998). The square is our data point obtained with the 2.2-m CAHA telescope and matches pretty well the expected value of $R_{\mathrm{c}}$ for this date. The point-dashed line is obtained when the data are fitted considering a $R_{\mathrm{c}}=25.5$ host galaxy $(\alpha=-2.12)$. The dotted line is the best fit when all the points and a host galaxy with $R=27.3$ are considered $(\alpha=-1.68)$. However, this fit is not satisfactory due to the presence of the $R_{\mathrm{c}}=25.5$ data point. Once excluded, the new fit is much better (with $\alpha=-1.78$, solid line). The long-dashed line is the best fit when no host galaxy is introduced $(\alpha=-1.70)$. A more extended discussion can be seen in Gorosabel (1999)

Acknowledgements. We are grateful to the entire BeppoSAX team for rapidly distributing the GRB positions, and to the Time Allocation Committees of the German-Spanish Calar Alto Observatorio and Observatorio del Teide (owned by the IAC). We also wish to thank the referee, L. Hanlon, for useful suggestions, and A. Bohm, K.J. Fricke, J. Greiner and K. Jäger for their help at some stage of this work.

\section{References}

Bloom J., et al., 1998a, GCN 30

Bloom J., et al., 1998b, ApJ 507, L25

Bloom J., Kulkarni S.R., 1998, GCN 161

Bond H., 1997, IAUC 6654

Castro-Tirado, et al., 1997, IAUC 6659

Castro-Tirado, et al., 1998a, IAUC 6848

Castro-Tirado, et al., 1998b, Sci 279, 1011

Castro-Tirado, et al., 1999, A\&SS (in press) (astroph/9903187)

Djorgovski G., et al., 1998, GCN 57

Galama T., et al., 1998, ApJ 497, L13

Gorosabel J., 1999, Ph.D. Thesis, Univ. of Valéncia

Groot, et al., 1998, ApJ 502, L123

Guarnieri A., et al., 1997, A\&A 328, L13

Pedersen, et al., 1998, ApJ 496, 311

Schechter P., 1976, ApJ 203, 297

Woosley S., 1999, A\&AS (this issue)

Zharikov S.V., et al., 1998, A\&A 337, 356 